\title{
Publisher's Note: Tunable Circularly Polarized Terahertz Radiation from Magnetized Gas Plasma [Phys. Rev. Lett. 114, 253901 (2015)]
}

W.-M. Wang, P. Gibbon, Z.-M. Sheng, and Y.-T. Li (Received 6 July 2015; published 21 July 2015)

DOI: $10.1103 /$ PhysRevLett.115.049902

PACS numbers: 42.65.Re, 52.38.-r, 32.80.Fb, 52.65.Rr, 99.10.Fg

This paper was published online on 23 June 2015 with an incorrect reference. Reference [18] should read as "Y. T. Li et al., Appl. Phys. Lett. 100, 254101 (2012); G. Q. Liao et al., Phys. Rev. Lett. 114, 255001 (2015)." The reference has been corrected as of 26 June 2015. The reference is correct in the printed version of the journal. 